\title{
Management of bone metastases: recent advances and current status
}

\author{
Kristopher Dennis • Vassilios Vassiliou • Tracy Balboni • \\ Edward Chow
}

Received: 24 May 2012 / Accepted: 6 July 2012 / Published online: 18 July 2012

(C) Springer-Verlag 2012

\begin{abstract}
This narrative review summarizes new and existing local and systemic management strategies for bone metastases from the fields of radiation oncology, medical oncology and orthopaedic surgery. Local management strategies are organized according to different clinical scenarios, with complicated bone metastases defined as those with associated pathological fractures, soft tissue components or those compressing the spinal cord or cauda equina, and uncomplicated lesions defined as those without these adverse features. The distinction applies for both sclerotic and lytic lesions. Systemic strategies are organized according to classes of therapeutic agents.
\end{abstract}

Keywords Bisphosphonates · Bone metastases ·

Chemotherapy $\cdot$ Radiotherapy $\cdot$ Surgery

\section{Introduction}

The skeleton is the most common site of metastatic disease. Autopsy work demonstrates bone metastases in well over two thirds of patients with breast and prostate carcinomas and over one third of patients with renal cell, thyroid, and

K. Dennis $\cdot$ E. Chow $(\bowtie)$

Department of Radiation Oncology, Odette Cancer Centre, Sunnybrook Health Sciences Centre, University of Toronto, 2075 Bayview Avenue,

Toronto, ON, Canada M4N3M5

e-mail: edward.chow@sunnybrook.ca

V. Vassiliou

Bank of Cyprus Oncology Centre,

Nicosia, Cyprus

T. Balboni

Department of Radiation Oncology, Dana-Farber/Brigham and Women's Hospital, Harvard Medical School,

Boston, MA, USA lung carcinomas [1,2]. Survival times following a diagnosis of bone metastases have increased substantially in recent years, especially for patients with breast and prostate primaries who routinely live for years with bone metastases. As such, these patients are at risk of developing skeletalrelated symptoms and complications over extended periods of time. Bone metastases can cause pain, reduce functional independence, induce hypercalcemia, compress neural structures such as the spinal cord and cauda equina, and compromise skeletal integrity leading to pathologic fractures. These events detract from quality of life, can delay anti-cancer therapies, are costly, and in certain settings can be life threatening.

\section{Uncomplicated bone metastases causing pain}

Pain is the most common symptom patients develop from bone metastases, and the gold standard therapy for its palliation remains conventional external beam radiotherapy (EBRT) [3, 4]. Radiotherapy can also restore and preserve physical functioning, provide local control, and maintain and improve skeletal integrity to reduce the risk of fracture. Dozens of trials have demonstrated that both single fraction (SF) and multiple fraction (MF) radiotherapy schedules reduce pain from previously unirradiated bone metastases. Typical schedules include 8 Gy in one fraction, 20 Gy in five fractions, $24 \mathrm{~Gy}$ in six fractions and $30 \mathrm{~Gy}$ in ten fractions. A recent meta-analysis of 25 trials comparing SF and MF schedules such as these showed similar overall pain response rates of $60 \%$ and $61 \%$, and complete pain response rates of $23 \%$ and $24 \%$ for SF and MF schedules, respectively [5]. MF schedules had a lower rate of retreatment than SF schedules (8\% vs $20 \%$ ), but many contend that the convenience and cost effectiveness of the SF schedule [6], the ability to safely and effectively re-irradiate if 
necessary, and the durability of the SF pain response [7] especially among patients with shorter life expectancies compensate for this shortcoming [8]. Authors of the guideline for palliative radiotherapy for bone metastases from the American Society for Radiation Oncology (ASTRO) could find no evidence to suggest that single 8 Gy fractions for either spinal or non-spinal bone metastases result in decreased rates of pain relief or increased rates of long-term toxicity [3].

However, repeated measures of practice patterns worldwide have shown that radiation oncologists more commonly administer MF schedules for uncomplicated bone metastases $[6,8]$. Factors that have been linked to a bias favoring MF schedules include: a non-academic practice setting, training within the USA, a US-based ASTRO membership $[6]$ and a fee-for service financial structure $[9,10]$. Given the expected rise in the prevalence of bone metastases within an aging population and the differential impact of SF and MF schedules on patients and third party payers, these factors deserve further study.

Standard treatment delivery recommendations for beam energies, arrangements and prescription points are provided by the International Consensus on Palliative Radiotherapy Endpoints for Future Clinical Trials in Bone Metastases which was recently updated [11]. For non-spine targets, one should prescribe to an isodose for single incident fields, and to mid-plane for opposed fields. For spine targets, one can prescribe to either the mid-vertebral body or anterior vertebral body for single incident fields. The American College of Radiology Appropriateness Criteria for nonspine bone metastases state that fluoroscopic simulation, computed tomography, and clinical simulation are all appropriate methods for planning these treatments. It further clarifies that there are no data showing an advantage for intensity-modulated radiation therapy, stereotactic body radiation therapy (SBRT), or proton therapy in this setting [4]. Similarly, the ASTRO guideline cautions that the delivery, efficacy, and safety of SBRT for uncomplicated bone metastases are still being established, and that SBRT should only be administered for this indication within a clinical trial [3].

The role of highly conformal radiotherapy in the treatment of bone metastases is an active area of research. Indeed, the steep dose gradients achievable with SBRT can allow for dose escalation and better normal tissue sparing compared to conventional EBRT. The Radiation Therapy Oncology Group 0631 Phase III study of image-guided radiosurgery/SBRT for localized spine metastases is currently randomizing patients with previously unirradiated spine metastases to either a control arm of $8 \mathrm{~Gy}$ in one fraction delivered with conventional external beam radiotherapy or an experimental arm of 16 or 18 Gy in one fraction delivered with radiosurgery/SBRT [12]. Patients are stratified according to the number of index spine metastases (1 vs. 2-3), the type of tumor (radioresistant vs. other) and the dose of radiosurgery/SBRT (16 vs. 18 Gy according to physician preference). The primary endpoint is pain control at the treated site(s) at 3 months following radiotherapy, and the investigators hypothesize that the experimental arm will result in a $40 \%$ improvement over the control arm in the proportion of patients experiencing pain relief. Other trials of SBRT in the de novo, re-irradiation and post-operative settings are anticipated or under development.

\section{Uncomplicated bone metastases in weight-bearing long bones at risk of fracture}

Metastatic lesions in weight-bearing long bones such as the femur can lead to pathologic fractures which cause significant morbidity and can ultimately shorten survival. Most high-risk lesions are lytic and initially produce microfractures that cause pain [1]; however, sclerotic lesions that meet certain criteria can be equally concerning [13]. Compared to reactive surgery following a fracture, prophylactic surgery quickly relieves pain, reduces trauma and operative blood loss, increases post-operative support-free ambulation, and results in shorter hospital admissions and more discharges to home rather than extended care facilities $[14,15]$. It also provides an opportunity for medical optimization prior to surgery which refines patient selection and minimizes perioperative morbidity.

Scoring systems have been created to predict the risk of fracture, so that prophylactic measures including surgery can be taken where appropriate. Harrington recommended prophylactic surgery to the femur if any of the following criteria are met: (1) a lesion of the proximal femur that is $\geq 2.5 \mathrm{~cm}$ in any dimension or is associated with an avulsion of the lesser trochanter, (2) a femoral lesion with $>50 \%$ of the circumferential cortical bone destroyed, or (3) if there is pain with weight bearing due to a femoral lesion that persists, increases or recurs following adequate radiotherapy [13]. Mirels' system, which is more commonly used, estimates the risk of a metastatic lesion causing a fracture by assigning it score out of 12 , based on four roentgenographic and clinical risk factors [16]. Lesions scoring a total of seven or less can be managed with radiotherapy alone, while those scoring eight or more require surgery (Table 1). This system has been found to have high sensitivity, but low specificity in subsequent validation studies [17]. Both Harrington's and Mirels' systems are valuable because they are easy to remember and they incorporate common and relevant clinical criteria, but they should only be used as guides and not rules, as they have been sparsely validated [17], have poor sensitivity [18] and omit other important factors that deserve consideration when contemplating surgery such as performance status, visceral metastases, comorbidities, predicted 
Table 1 Mirels' scoring system for diagnosing impending pathological fractures ${ }^{16}$

\begin{tabular}{llll}
\hline Variable & \multicolumn{3}{l}{ Score } \\
\cline { 2 - 4 } & 1 & 2 & 3 \\
\hline Site & Upper limb & Lower limb & Peritrochanter \\
Pain & Mild & Moderate & Functional \\
Lesion & Blastic & Mixed & Lytic \\
Size & $<1 / 3$ diameter & $1 / 3-2 / 3$ diameter & $>2 / 3$ diameter
\end{tabular}

Bone metastases with a total score $\leq 7$ can be managed with radiotherapy alone while those $\geq 8$ require surgery

duration of recovery, life expectancy, quality of surrounding unaffected bone, and inter- and intraobserver variability in score assignment $[17,19]$. The potential complications of surgery must also be considered. These include fat and tumor emboli, infections, hematomas, late dislocations and failed fixations [19].

Impending or outright fractures of the femoral head, femoral neck, or the intertrochanteric region can be treated with hemiarthroplasty. If the acetabulum is involved then total hip arthroplasty is preferred, often with cement augmentation. Intramedullary nailing is indicated if the diaphysis or distal femur is also involved. Extramedullary fixation of periarticular lesions with plates and screws is also appropriate, and cement augmentation is useful here as well $[15,19]$.

Following surgery, post-operative radiotherapy is indicated to eradicate remaining microscopic disease, control potentially remaining macroscopic disease, promote bone healing, encourage normal limb function, and reduce the need for repeat surgery [20]. However, the ability of radiotherapy to promote new bone formation around surgical implants has been questioned by a study that showed better results in unirradiated rather than radiated patients post-operatively [21]. If normal tissue constraints allow, the entire surgical implant should be included within the radiotherapy volume, and MF schedules are most commonly administered. No studies have compared different post-operative treatment volumes, different timing delays from the time of surgery or different dose fractionation schedules, and no single fraction postoperative reports have been published. For patients with high-risk lesions that cannot undergo surgery, an MF schedule is indicated to reduce pain, promote mineralisation, and decrease the risk of fracture [4, 22]. Nonpainful lesions that still pose a risk of fracture according to other criteria should still be irradiated.

Future studies need to refine fracture risk estimates through the use of CT or MRI-based algorithms that can quantify bone structural integrity [17]. Successful new models will need to be non-invasive, practical, affordable and prospectively validated.

\section{Pathological fractures of long bones}

Surgical procedures as described above should always be considered for fractures that impede function or cause mechanical pain, even in patients with short life expectancies. Radiotherapy alone cannot restore skeletal stability, and it is generally ineffective at palliating mechanical pain. Postoperative radiotherapy should be administered after surgery as described earlier.

\section{Complicated bone metastases with pathological compression fractures and/or soft tissue components}

Although pathological fractures of weight-bearing long bones rightly garner much attention, fractures of axial skeleton components such as the vertebrae and ribs are actually more prevalent [1] and can lead to significant morbidity. Likewise, advanced lesions with soft tissue components extending beyond the normal cortical boundaries of involved bones can be problematic, especially when they encroach upon neural structures. Neuropathic pain is common in this setting and is particularly difficult to treat, despite advances in adjuvant non-opioid analgesics.

Skeletal instability can lead to mechanical pain in these lesions. This is best addressed through operative or interventional procedures in fit patients, with consideration of fractionated consolidative radiotherapy afterwards. During such procedures, fractures can be stabilized, bone can be reconstructed and critical structures can be decompressed [19]. Although pain relief is still the most important goal of treatment, long-term skeletal stability and local tumor control are also desirable depending on the performance status and life expectancy of the patient. Mechanical pain is poorly palliated with radiotherapy alone regardless of the dose, but when other measures are not possible, MF schedules are generally favored due to the belief that a dose response exists for these lesions. Unfortunately, most previous trials that compared SF and MF schedules had no threedimensional radiologic review, and the proportion of lesions irradiated in those trials that were by definition complicated is unknown. One unique retrospective report of 603 patients with spinal metastases treated with radiotherapy at a single institution categorized patients as having either mass type $(n=111)$ or non-mass type $(n=492)$ tumors, with mass type defined as those with a clear boundary outside the vertebra [23]. Most patients were evaluated with three-dimensional computed tomography findings used in treatment planning. On multivariate analysis, a non-mass type tumor was associated with better local control, with local recurrence defined as either regrowth of the tumor within the irradiated field (by CT or MRI) or exacerbation of pain or motor deficits in a previously irradiated spinal region. No 
breakdown of these events was provided, but the authors reasonably concluded that mass-type tumors require higher dose radiotherapy than non-mass-type tumors. Indeed, there is great interest in evaluating single and multiple fraction SBRT with standardized endpoints for pain relief and local control for complicated vertebral lesions in both the de novo and post-operative settings. At present, reports are limited to a few phase II studies with limited follow-up and retrospective single institution reviews $[15,24]$.

\section{Complicated bone metastases causing compression of the spinal cord and/or cauda equina}

Spinal cord and cauda equina compression resulting from bone metastases are emergencies that require prompt multidisciplinary care [25]. A population-based study of the Canadian province of Ontario during 1990-1995 reported that $2.5 \%(n=3,458)$ of all cancer patients who died from their disease had been admitted at least once for malignant spinal cord compression [26]. Incidence rates ranged from $7.9 \%$ in patients with myeloma to $0.2 \%$ in patients with pancreatic cancer. The thoracic spine is affected in approximately $70 \%$ of cases of malignant extradural spinal cord compression (MESCC), followed by the lumbar (20\%) and cervical spines $(10 \%)[19,27]$; however, multi-level compression is also not uncommon.

A systematic review and management guideline for MESCC has recently been updated which defines the roles of steroids, surgery and radiotherapy [28]. For patients with neurologic deficits, steroids are recommended if MESCC is confirmed or suspected. Doses administered within the literature vary widely, and patients must always be monitored for steroid toxicity. The authors recommended an initial dexamethasone bolus of $8-10 \mathrm{mg}$ followed by $16 \mathrm{mg}$ daily as was done within two randomized phase III radiotherapy-only trials for MESCC [29, 30]. This maintenance dose is also recommended in the NICE metastatic spinal cord compression guideline [25]. Alternatively, for patients with dense paraparesis, an initial bolus of $100 \mathrm{mg}$ followed by $96 \mathrm{mg}$ daily could be considered [28], similar to what was done within a randomized phase III trial of surgery and radiotherapy vs. radiotherapy alone [31], and a 20-patient feasibility trial that randomized patients to either 96 or $16 \mathrm{mg}$ daily [32].

Surgical opinions should be sought early [25], as advances in the field now provide many options with differing recovery times [19]. Surgery is effective in decompressing and stabilizing the spinal cord, nerve roots and vertebral column. Surgery followed by fractionated radiotherapy should be considered [ 3 , 28] for patients with a single level of compression [31], especially those with bony compression or spinal instability, provided that the patient is medically fit, surgically operable and has a good performance status [3, 28]. Indeed, a randomized phase III of 101 patients showed that compared to patients treated with radiotherapy alone, those treated with decompressive surgery followed by post-operative radiotherapy were significantly more likely to regain or retain ambulatory ability [31]. Notably, a recent matched pair analysis of 324 patients with MESCC managed with either surgery followed by radiotherapy or radiotherapy alone reported no difference in outcomes between groups [33]. This suggests there may be a population of MESCC patients for whom surgery may be omitted, although further studies are required to refine best practice approaches. Patients with compression caused by bone alone and those whose symptoms have developed slowly and not resulted in complete deficits for longer than $12-24 \mathrm{~h}$ are the most likely to benefit from surgery [19]. Evidence does not support the use of surgery alone at this time, however [3, 4].

Patients receiving radiotherapy alone are typically managed with fractionated schedules such as 20 Gy in five fractions or $30 \mathrm{~Gy}$ in ten fractions [3, 28]. However, patients with limited life expectancy should receive a single fraction of $8 \mathrm{~Gy}$ [28], as a randomized phase III trial of 327 patients with a poor prognosis (defined according to poor histologic features and/ or poor performance status or functional impairment) showed no difference between a control arm of $16 \mathrm{~Gy}$ in two fractions over 1 week and an experimental arm of $8 \mathrm{~Gy}$ in one fraction for the endpoints of ambulation, duration of ambulation, bladder control, pain response and overall survival [30]. Ongoing trials may further define the role of single fraction radiotherapy in patients with better prognoses [34] treated with radiotherapy alone, and its role in the post-operative setting needs to be determined as well $[3,34]$.

\section{Recurrent pain following radiotherapy}

Irradiated bone metastases can require retreatment due to insufficient pain control, pain recurrence or local progression. Pooled results from 12 phase III trials of conventional external beam radiotherapy showed an $8 \%$ rate of retreatment for lesions previously treated with MF schedules and a $20 \%$ rate for lesions previously treated with SF schedules [5]. An additional recent systematic review and meta-analysis reported a $58 \%$ pain response rate following re-irradiation [35]. Patients initially treated with either SF or MF schedules can derive pain relief from reirradiation. It can be delivered via either SF or MF schedules; however, the optimal dose fractionation remains unknown. However, a large international phase III equivalency trial that randomizes patients to either SF or MF re-irradiation is ongoing and should report its findings in the coming year [36]. SBRT will likely have an important role to play in the future, as single institution reports of spinal reirradiation with SBRT have demonstrated that it can be delivered safely and effectively [37-39]. 


\section{Solitary or oligometastases}

Solitary bone lesions often require thorough investigation, especially in patients with no oncologic history. Primary and secondary osseous tumors both need to be considered as their treatments differ greatly. Biopsies planned following multidisciplinary meetings can often secure the diagnosis. Oligometastatic osseous lesions are currently the focus of great interest, with single and multiple fraction SBRT schedules potentially holding the promise of cure. Patients fitting the oligometastatic phenotype should ideally be treated within clinical trials, as evidence confirming or refuting the curative promise is still developing.

\section{Bisphosphonates}

Bisphosphonate (Bp) use has increased dramatically in the last decade, becoming an integral part of the oncologic management of patients with skeletal metastases [40]. Currently, three generations of Bps are known, with clodronate belonging to the first generation of compounds with a weak antiresorptive activity [41]. Second-generation Bps have an intermediate potency, and typical examples are pamidronate and ibandronate [42]. The most potent Bps are the third generation compounds, with zoledronate being the most well-known representative. The relative potency of clodronate to ibandronate and zoledronate is 1:857:16,700 [43].

After intravenous Bp administration, 25-40\% of the injected dose is excreted through the kidney, with the remaining binding avidly to the bone matrix where it is deposited for months or even years [15]. Bps concentrate selectively on bone resorption surfaces where their concentrations range between 0.1 to $1 \mathrm{mM}$ [44]. Osteoclasts take up Bps by active endocytosis causing a reduction of their activity and viability through apoptosis $[45,46]$.

Bps are effective at reducing the risk of skeletal related events (SREs) in patients with bone metastases from solid tumors and myeloma [47-59]. They have also been shown to be effective in reducing skeletal pain $[47,60,61]$ and maintaining or even improving the quality of life of treated patients $[60,62,63]$.

\section{Bisphosphonate treatment according to primary tumor}

\section{Breast cancer}

In breast cancer, both oral (ibandronate and clodronate) and i.v. Bps have shown efficacy $[64,65]$. In a large meta-analysis by Wong et al., cochrane data showed that Bps reduce the SRE risk by $15 \%$ (risk ratio (RR) 0.85 ), with the RR risk for zoledronic acid being 0.59 , pamidronate 0.77 and i.v. ibandronate 0.80 [66]. A considerable delay in the median time to SREs was also reported, as well as significantly improved bone pain in six out of 11 studies [66].

\section{Prostate cancer}

Zoledronic acid has shown a statistically significant longterm reduction in the SRE risk as compared to placebo, in patients with hormone refractory prostate cancer [67, 68]. The proportion of patients with an on-study SRE was reduced by $22 \%$, and the median time to SREs was delayed by more than 5 months. A reduction of the ongoing risk for SREs by $36 \%$ was also reported $[67,68]$.

BPs in other solid tumors

In a study by Rosen et al., patients with bone metastases from non-small cell lung carcinoma or other solid tumors were randomized to receive either zoledronic acid or placebo. Treatment with zoledronic acid brought about a $31 \%$ significantly lower risk for developing an SRE and a significantly longer median time to the development of the first SRE [52]. Additionally, a long-term subset analysis of this study involving patients with renal cell carcinoma showed that zoledronic acid achieved a reduction in the SRE incidence by $41 \%$ [69].

\section{Concurrent application of bisphosphonates and radiotherapy}

The concurrent application of RT and Bps has shown to bring about a significant pain response, enhanced reossification and improved quality of life in patients with bone metastases from a variety of solid tumors [70-73]. This is a result of an additive and synergistic effect of the two treatment modalities on osteoclast function $[70,72]$.

\section{RANK ligand inhibitors}

RANK ligand (RANKL) inhibitors provide a targeted approach that stops the vicious cycle leading to bone lysis and destruction. RANKL inhibition has decreased osteolytic and osteoblastic skeletal changes and associated tumor progression in several animal models of cancer and bone metastases including breast, prostate, lung and multiple myeloma [74-79].

Denosumab is a RANKL inhibitor that is approved in the USA for preventing SREs in patients with metastatic bone disease from solid tumors. It is a fully human monoclonal antibody that specifically binds to the RANKL receptor. This inhibits osteoclast activity and bone turnover to a 
similar or greater extent as compared to i.v. Bps $[80,81]$. The optimal dosing regimen has been defined in phase II trials and $120 \mathrm{mg}$ is administered subcutaneously every 4 weeks.

Three double-blind studies have evaluated the effectiveness of denosumab vs. zoledronate in patients with breast, prostate or other solid tumors and multiple myeloma [82-84]. The primary end point was defined as the time to first on-study SRE. In patients with breast or castrateresistant prostate cancer denosumab delayed the time to first SRE by $18 \%$ as compared to zoledronic acid and in patients with solid tumors or multiple myeloma the delay was $16 \%$ [82-84]. It has also been reported to prevent treatmentinduced bone loss and pathologic fractures associated with androgen deprivation therapy [85].

\section{Radionuclide therapy}

Bone-seeking radiopharmaceuticals (BSRs) are especially useful in patients with widespread skeletal lesions, providing selective delivery of ionizing radiation to targeted areas of increased osteoblast activity [42]. Intravenous injection of strontium-89 (Sr-89), samarium-153 (Sm-153), and rhenium-186 (Re-186) are approved for the treatment of skeletal pain resulting from either sclerotic or mixed bone lesions from prostate and breast carcinomas or any other tumor. Osteoblastic lesions should be evident on whole body skeletal scintigraphy [86-89].

Even though BSRs achieved significant overall pain responses that ranged between $57 \%$ and $92 \%$, they have not gained wide acceptance in the oncologic community. Most patients treated with BSRs have already developed disease progression while on chemotherapy or other pharmacologic treatments, or they are not candidates for external beam radiotherapy due to previous irradiation or limiting potential side effects [90]. This paradigm should be avoided since the early application of BSRs has been shown to be safe and effective in managing bone pain [91]. The main potential side effects of BSRs are a transient pain flare reaction that lasts for about $72 \mathrm{~h}$ and transient myelosuppression that affects mainly platelets and white blood cells [90]. A recent study by Kurosaka et al. showed that apart from pain relief, treatment with BSRs results in significant improvement in quality of life [92].

\section{Bone-seeking radiopharmaceuticals and chemotherapy}

A potential advantage of the concurrent administration of BSRs and chemotherapy is improved efficacy that may be achieved by chemo-sensitization of tumor cells. This is a wellknown effect that renders tumor cells more susceptible to radiation emitted by BSRs [90]. A few studies investigating their combination have reported promising results. In a study involving patients with painful bone metastases, low-dose carboplatin was combined with Sr-89. A pain response was evident in $74 \%$ of patients, with those receiving combined treatment having a statistically increased response as compared to those receiving Sr-89 alone. There was also marginally better survival in the group receiving combined treatment. No significant toxicity was evident in any group [93].

In a different phase II trial involving patients with hormone refractory prostate carcinoma, induction chemotherapy was initially applied, followed by either doxorubicin with or without $\mathrm{Sr}-89$ in the case that there was stable or responsive disease [94]. Overall, $60 \%$ of patients had a decrease in their serum prostate-specific antigen (PSA) level, and almost $52 \%$ of patients had a complete pain response. Median survival was also significantly superior for patients on combined treatment ( 27.7 vs 16.8 months) ( $p=0.0014$ ).

A renewed interest was seen recently with two studies investigating the effectiveness of the combination of BSRs and docetaxel in patients with castration-resistant prostate carcinoma $[95,96]$. In one of these studies by Fizazi et al., patients achieving stabilization following four cycles of docetaxel and estamustine were further treated with combined docetaxel and $\mathrm{Sm}-153$. A PSA response was seen in $77 \%$ of patients and pain relief in $69 \%$. Combined treatment was well tolerated and a median survival of 29 months was achieved [96].

Even though the above results are promising, current clinical practice does not recommend the concurrent application of BSRs and chemotherapy outside of clinical trials [97].

\section{Bone-seeking radiopharmaceuticals and bisphosphonates}

Both BSRs and Bps are effective at reducing skeletal pain due to metastatic bone disease. Their concurrent application is rather controversial due to the possibility of a competitive interaction at the hydroxyapatite crystal bone surface [90], although a few studies that evaluated their concurrent application yielded promising results.

In a pivotal study by Storto et al., patients with bone metastases from prostate or breast cancer were managed with either six infusions of zoledronic acid every 3 weeks, followed by a single dose of $\mathrm{Sr}-89$ (group A), or $\mathrm{Sr}-89$ alone (group B), or zoledronate alone for 8 months (group C). A statistically significant reduction in pain score was noted for all groups, with group A patients experiencing a more pronounced response $(p<0.001)$. Moreover, $68 \%$ of patients from group A achieved a pain response $\geq 4$ points as compared to $15 \%$ and $9 \%$ for groups B and C, respectively [98]. 
Re-186 was administered concurrently with pamidronate in a study involving breast cancer patients and bone metastases. In this study, it was shown that Bp use did not interfere with the BSR uptake or effect. Additionally, pain response was similar and statistically significant for both groups [99]. In a further study involving breast cancer patients with metastatic bone lesions, patients received either Re-188 (group A), pamidronate alone (group B) or combined treatment (group C) [100]. Good pain responses were seen, with the corresponding rates being $73.3 \%, 80 \%$ and $100 \%$ for groups $\mathrm{A}-\mathrm{C}$, respectively. The investigators concluded that the combined treatment achieved a significantly better therapeutic effect $(p<0.05)$ [100].

\section{Health-related quality of life}

Bone metastases and their treatments can negatively impact health-related quality of life (HRQOL), and new therapies need to be evaluated with respect to this important subjective and multidimensional construct. Recent developments include the creation of the EORTC-QLQ$\mathrm{BM} 22$, a 22-item questionnaire that was designed to specifically address the clinically meaningful factors that influence HRQOL among patients with bone metastases [101]. It is intended to be used in conjunction with the EORTC-QLQ-30 general quality of life questionnaire. The EORTC-QLQ-BM22 has undergone international field testing and validation and is available for use in clinical trials. The older 16-item Functional Assessment of Cancer Therapy-Bone Pain questionnaire is the only other tool that focuses on bone metastases-specific issues to the same degree [102].

\section{Conclusions}

In summary, bone metastases are a frequent complication of advanced cancers, and they worsen patient quality of life due to their propensity to cause symptoms and other complications. Radiotherapeutic, surgical, interventional and systemic treatment options should be carefully considered and applied promptly to relieve symptoms and preserve or improve quality of life. Management strategies should be personalized and address the characteristics of the bone metastases at hand while respecting the larger picture of the patient's trajectory of illness. Further research of best practices is required to advance the evidence base guiding these strategies. Methodologies should be rigorous, but still accommodate patients with varying life expectancies. Evaluation of health-related quality of life using validated instruments is of paramount importance.
Conflicts of interest The authors declare no relevant conflicts of interest.

\section{References}

1. Coleman RE (2006) Clinical features of metastatic bone disease and risk of skeletal morbidity. Clin Cancer Res 12(20 suppl):6243-6249

2. Blum RH, Novetsky D, Shasha D et al (2003) The multidisciplinary approach to bone metastases. Oncology 17(6):1-18

3. Lutz S, Berk L, Chang E et al (2011) Palliative radiotherapy for bone metastases: an ASTRO evidence-based guideline. Int J Radiat Oncol Biol Phys 79(4):965-976

4. Lutz ST, Lo SSM, Chang EL et al (2012) ACR Appropriateness Criteria non-spine bone metastases. J Palliat Med 15(5):1-6

5. Chow E, Zeng L, Salvo N et al (2012) Update on the systematic review of palliative radiotherapy trials for bone metastases. Clin Oncol (R Coll Radiol) 24:112-124

6. Fairchild A, Barnes E, Shosh S et al (2009) International patterns of practice in palliative radiotherapy for painful bone metastases: evidence-based practice? Int J Radiat Oncol Biol Phys 75 (5):1501-1510

7. van der Linden Y, Steenland E, van Houwelingen HC et al (2006) Patients with a favourable prognosis are equally palliated with single and multiple fraction radiotherapy: results on survival in the Dutch Bone Metastases Study. Radiother Oncol 78:245-253

8. Chow E, Hahn CA, Lutz ST (2012) Global reluctance to practice evidence-based medicine continues in the treatment of uncomplicated painful bone metastases despite level 1 evidence and practice guidelines. Int J Radiat Oncol Biol Phys 83(1):1-2

9. Lievens Y, Van den Bogaert W, Rijnders A (2000) Palliative radiotherapy practice within Western European countries: impact of the radiotherapy financing system? Radiother Oncol 56 (3):289-295

10. Kachnic L, Berk L (2005) Palliative single-fraction radiation therapy: how much more evidence is needed? J Natl Canc Inst 97(11):786-788

11. Chow E, Hoskin P, Mitera G et al (2012) Update of the international consensus on palliative radiotherapy endpoints for future clinical trials in bone metastases. Int J Radiat Oncol Biol Phys 82 (5): 1730-1737

12. RTOG 0631 Protocol Information. http://www.rtog.org/ ClinicalTrials/ProtocolTable/StudyDetails. aspx? study $=0631$. Accessed 15 May 2012

13. Harrington KD (1986) Impending pathologic fractures form metastatic malignancy: evaluation and management. Instr Course Lect 35:357-381

14. Ward WG, Holsenbeck S, Dorey FJ (2003) Metastatic disease of the femur: surgical treatment. Clin Orthop Relat Res 415(Suppl): S230-S244

15. Chow E, Finkelstein JA, Coleman RE (2008) Metastatic cancer to bone. In: DeVita VT, Lawrence TS, Rosenberg SA (eds) Cancer: principles and practice of oncology, 8th edn. Lippincott Williams and Wilkins, Philadelphia, pp 2510-2519

16. Mirels H (1989) Metastatic disease in long bones: a proposed scoring system for diagnosing impending pathologic fractures. Clin Orthop Relat Res 249:256-264

17. Attar S, Steffner RJ, Avedian R et al (2012) Surgical intervention of nonvertebral osseous metastasis. Canc Control 19(2):113-121

18. Damron TA, Ward WG (2003) Risk of pathologic fracture: assessment. Clin Orthop 415(Suppl):S208-S211

19. Eastley N, Newey M, Ashford RU (2012) Skeletal metastasesthe role of the orthopaedic and spinal surgeon. Surg Oncol doi:10.1016/j.suronc.2012.04.001 
20. Townsend PW, Smalley SR, Cozad SC et al (1995) Role of postoperative radiation therapy after stabilization of fractures caused by metastatic disease. Int J Radiat Oncol Biol Phys 31 (1):43-49

21. Haentjens P, De Neve W, Opdecam P (1995) Prosthesis for the treatment of metastatic bone disease of the hip: effects of radiotherapy. Bull Du Canc 82:961-970

22. van der Linden YM, Kroon HM, Dijkstra SP (2003) Simple radiographic parameter predicts fracturing in metastatic femoral bone lesions: results from a randomised trial. Radiother Oncol 69 (1):21-31

23. Mizumoto M, Harada H, Asakura $H$ et al (2011) Radiotherapy for patients with metastases to the spinal column: a review of 603 patients at Shizuoka Cancer Center Hospital. Int J Radiat Oncol Biol Phys 79(1):208-213

24. Sahgal A, Larson DA, Chang EL (2008) Stereotactic body radiosurgery for spinal metastases: a critical review. Int J Radiat Oncol Biol Phys 71(3):652-665

25. National Institute for Health and Clinical Excellence. Metastatic spinal cord compression. Diagnosis and management of adults at risk of and with metastatic spinal cord compression. Issue date November 2008. http://www.nice.org.uk/nicemedia/live/12085/ 42653/42653.pdf. Accessed 20 May 2012

26. Loblaw CA, Laperriere NJ, Mackillop WJ (2003) A populationbased study of malignant spinal cord compression in Ontario. Clin Oncol (R Coll Radiol) 15(4):211-217

27. Klimo P Jr, Schmidt MH (2004) Surgical management of spinal metastases. Oncologist 9(2):188-196

28. Loblaw DA, Mitera D, Ford M et al (2012) A 2011 Updated systematic review and clinical practice guideline for the management of malignant extradural spinal cord compression. Int $\mathrm{J}$ Radiat Oncol Biol Phys Mar 13 [Epub ahead of print]

29. Maranzano E, Bellavita R, Rossi R (2005) Short-course versus split-course radiotherapy in metastatic spinal cord compression: results of a phase III, randomized, multicenter trial. J Clin Oncol 23(15):3358-3365

30. Maranzano E, Trippa F, Casale M et al (2009) 8Gy single-dose radiotherapy is effective in metastatic spinal cord compression: results of a phase III randomized multicentre Italian trial. Radiother Oncol 93(2):174-179

31. Patchell RA, Tibbs PA, Regine WF et al (2005) Direct decompressive surgical resection in the treatment of spinal cord compression caused by metastatic cancer: a randomised trial. Lancet 366(9486):643-648

32. Graham PH, Capp A, Delaney G et al (2006) A pilot randomised comparison of dexamethasone $96 \mathrm{mg}$ vs $16 \mathrm{mg}$ per day for malignant spinal-cord compression treated by radiotherapy: TROG 01.05 Superdex study. Clin Oncol (R Coll Radiol) 18(1):70-76

33. Rades D, Huttenlocher S, Dunst J et al (2010) Matched pair analysis comparing surgery followed by radiotherapy and radiotherapy alone for metastatic spinal cord compression. J Clin Oncol 28(22):3597-3604

34. Holt T, Hoskin P, Maranzano E et al (2012) Malignant epidural spinal cord compression: the role of external beam radiotherapy. Curr Opin Support Palliat Care 6(1):103-108

35. Huisman M, van den Bosch MA, Wijlemans JW et al (2012) Effectiveness of reirradiation for painful bone metastases: a systematic review and meta-analysis. Int J Radiat Oncol Biol Phys doi:10.1016/j.ijrobp.2011.10.080

36. Chow E, Hoskin PJ, Wu J et al (2006) A phase III international randomized trial comparing single with multiple fractions for reirradiation of painful bone metastases: National Cancer Institute of Canada Clinical Trials Group (NCIC CTG) SC 20. Clin Oncol (R Coll Radiol) 18(2):125-128

37. Sahgal A, Ames C, Chou D et al (2009) Stereotactic body radiotherapy is effective salvage therapy for patients with prior radiation of spinal metastases. Int J Radiat Oncol Biol Phys 74 (3):723-731

38. Garg AK, Wang XS, Shiu AS et al (2011) Prospective evaluation of spinal reirradiation by using stereotactic body radiation therapy: the University of Texas MD Anderson Cancer Center experience. Cancer 117(15):3509-3516

39. Sahgal A, Ma L, Weinberg V et al (2012) Reirradiation human spinal cord tolerance for stereotactic body radiotherapy. Int $\mathrm{J}$ Radiat Oncol Biol Phys 82(1):107-116

40. Vassiliou V, Kardamakis D (2009) The management of metastatic bone disease with the combination of bisphosphonates and radiotherapy: from theory to clinical practice. Anticanc Agents Med Chem 9:326-335

41. Rogers MJ, Gordon S, Benford HL et al (2000) Cellular and molecular mechanisms of action of bisphosphonates. Cancer 88: S2961-S2978

42. Vasiliou V, Bruland O, Janjan N et al (2009) Combining systemic bisphosphonates with palliative external been radiotherapy or bone-targeted radionuclide therapy: interactions and effectiveness. Clin Oncol 21:665-667

43. Green JR, Muller K, Jaeggi KAJ (1994) Preclinical pharmacology of CGP 42'446, a new, potent, hetero cyclic bisphosphonate compound. Bone Miner Res 9:745-751

44. Sato M, Grasser W, Endo N et al (1991) Bisphosphonate action. Aledronate localization in rat bone and effects on osteoclast ultrastructure. Clin Invest 88:2095-2105

45. Russell RG, Rogers MJ (1999) Bisphosphonates: from the laboratory to the clinic and back again. Bone 25:97-106

46. Rogers MJ, Brown RJ, Hodkin V et al (1996) Bisphosphonates are incorporated into adenine nucleotides by human aminoacyltRNA synthetase enzymes. Biochem Biophys Res Commun 224:863-869

47. Body JJ, Diel IJ, Lichinister MR et al (2003) Intravenous ibandronate reduces the incidence of skeletal complications in patients with breast cancer and bone metastases. Ann Oncol 14:1399-1405

48. Pavlakis N, Stocker M (2002) In the Conchrane library: bisphosphonates for breast cancer. Issue 1; Oxford: update software

49. Body JJ, Diel IJ, Lichinitser M et al (2004) Oral ibandronate reduces the risk of skeletal complications in breast cancer patients with metastatic bone disease: Results from two randomized, placebo-controlled phase III studies. Br J Cancer 90:1133-1137

50. Hortobagyi GN, Theriault R, Lipton A et al (1998) Long-term prevention of skeletal complications of metastatic breast cancer with pamidronate. J Clin Oncol 16:2038-2044

51. Khono N, Aogi K, Minami H et al (2005) Zoledronic acid significantly reduces skeletal complications compared with placebo in Japanese women with bone metastases from breast cancer: a randomized, placebo-controlled trial. J Clin Oncol 23:3314-3321

52. Rosen L, Gordon D, Tchekmedyian S et al (2003) Zoledronic acid versus placebo in the treatment of skeletal metastases in patients with lung cancer and other solid tumors: a phase III, double-blind, randomized trial-the Zoledronic Acid Lung Cancer and Other Solid Tumors Study Group. J Clin Oncol 2:3150-3157

53. Brown JE, Neville-Webbe H, Coleman RE (2004) The role of bisphosphonates in breast and prostate cancers. Endocr Relat Canc 11:207-224

54. Berenson JR, Lichtenstein A, Porter L et al (1996) Efficacy of pamidronate in reducing skeletal events in patients with advanced multiple myeloma. Myeloma Aredia Study Group. N Engl J Med 334:488-493

55. Berenson JR, Lichtenstein A, Porter L et al (1998) Long-term pamidronate treatment of advanced multiple myeloma patients reduces skeletal events. Myeloma Aredia Study Group. J Clin Oncol 16:593-602 
56. Rosen LS, Gordon D, Kaminski M et al (2001) Zoledronic acid versus pamidronate in the treatment of skeletal metastases in patients with breast cancer or osteolytic lesions of multiple myeloma: a phase III, double-blind, comparative trial. Canc J 7:377387

57. Rosen LS, Gordon D, Kaminski M et al (2003) Long-term efficacy and safety of zoledronic acid compared with pamidronate disodium in the treatment of skeletal complications in patients with advanced multiple myeloma or breast carcinoma: a randomized, double-blind, multicenter, comparative trial. Cancer 98:1735-1744

58. Berenson JR, Hillner BE, Kyle RA et al (2002) American Society of Clinical Oncology clinical practice guidelines: the role of bisphosphonates in multiple myeloma. J Clin Oncol 20:37193736

59. Kyle RA, Yee GC, Somerfield MR et al (2007) American Society of Clinical Oncology 2007 clinical practice guideline update on the role of bisphosphonates in multiple myeloma. J Clin Oncol 25:2464-2472

60. Body JJ, Diel IJ, Bell R et al (2004) Oral ibandronate improves bone pain and preserves quality of life in patients with skeletal metastases due to breast cancer. Pain 111:306-312

61. Gralow J, Tripathy DJ (2007) Managing metastatic bone pain: the role of bisphosphonates. Pain Symptom Manag 33:462-472

62. Diel IJ, Body JJ, Lichinitser MR et al (2004) Improved quality of life after long-term treatment with the bisphosphonate ibandronate in patients with metastatic bone disease due to breast cancer. Eur J Cancer 40:1704-1712

63. Diel IJ (2007) Effectiveness of bisphosphonates on bone pain and quality of life in breast cancer patients with metastatic bone disease; a review. Support Care Canc 15:1243-1249

64. Coleman RE (2008) Risks and benefits of bisphosphonates. Br J Cancer 98:1736-1740

65. Pavlakis N, Schmidt R, Stockler M (2005) Bisphosphonates for Breast Cancer. Cochrane Database Syst Rev 3:CD003474

66. Wong MH, Stockler MR, Pavlakis N (2012) Bisphosphonates and other bone agents for breast cancer. Cochrane Database Syst Rev 2:CD033474

67. Saad F, Gleason DM, Murray R et al (2002) A randomized, placebo-controlled trial of zoledronic acid in patients with hormone-refractory metastatic prostate carcinoma. J Natl Canc Inst 94:1458-1468

68. Saad F, Gleason DM, Murray R et al (2004) Long-term efficacy of zoledronic acid for the prevention of skeletal complications in patients with metastatic hormone-refractory prostate cancer. J Natl Canc Inst 96:879-882

69. Lipton A, Seaman J, Zheg M. Long-term efficacy and safety of zoledronic acid in patients with bone metastases from renal cell carcinoma. Presented at: what is now in bisphosphonates? Seventh workshop on bisphosphonates - from the laboratory to the patient, March 24-26, 2004, Davos, Switzerland

70. Vassiliou V, Kalogeropoulou C, Christopoulos C et al (2007) Combination ibandronate and radiotherapy for the treatment of bone metastases: clinical evaluation and radiologic assessment. Int J Radiat Oncol Biol Phys 67:264-272

71. Vassiliou V, Kalogeropoulou C, Giannopoulou E et al (2007) A novel study investigating the therapeutic outcome of patients with lytic, mixed and sclerotic bone metastases treated with combined radiotherapy and ibandronate. Clin Exp Metastasis 24:169-178

72. Kouloulias V, Matsopoulos G, Kouvaris J et al (2003) Radiotherapy in conjunction with intravenous infusion of $180 \mathrm{mg}$ of disodium pamidronate in management of osteolytic metastases from breast cancer: clinical evaluation, biochemical markers, quality of life, and monitoring of recalcification using assessment of greylevel histogram in plain radiographs. Int J Radiat Oncol Biol Phys $57: 143-157$
73. Vassiliou V, Leotsinides M, Kalogeropoulou C et al (2009) Concurrent application of bisphosphonates and external beam radiotherapy in patients with metastatic bone disease from renal cancer. BJU Int 103:620-624

74. Armstrong AP, Miller RE, Jones JC et al (2008) RANKL acts directly on RANK-expressing prostate tumor cells and mediates migration and expression of tumor metastasis genes. Prostate 68:92-104

75. Buijs JT, Que I, Lowik CW et al (2009) Inhibition of bone resorption and growth of breast cancer in the bone microenvironment. Bone 44:380-386

76. Canon JR, Roudier M, Bryant R et al (2008) Inhibition of RANKL blocks skeletal tumor progression and improves survival in a mouse model of breast cancer bone metastasis. Clin Exp Metastasis 25:119-129

77. Feeley BT, Liu NQ, Conduah AH et al (2006) Mixed metastatic lung cancer lesions in bone are inhibited by noggin overexpression and Rank:Fc administration. J Bone Miner Res 21:15711580

78. Pearse RN, Sordillo EM, Yaccoby S et al (2001) Multiple myeloma disrupts the TRANCE/osteoprotegerin cytokine axis to trigger bone destruction and promote tumor progression. Proc Natl Acad Sci U S A 98:11581-11586

79. Zheng Y, Zhou H, Brennan K et al (2007) Inhibition of bone resorption, rather than direct cytotoxicity, mediates the antitumour actions of ibandronate and osteoprotegerin in a murine model of breast cancer bone metastasis. Bone 40:471-478

80. Canon J, Bryant R, Roudier M et al (2010) Inhibition of RANKL increases the antitumor effect of the EGFR inhibitor panitumumab in a murine model of bone metastasis. Bone 46:1613-1619

81. Holland PM, Miller R, Jones J et al (2010) Combined therapy with the RANKL inhibitor RANK-Fc and rhApo2L/TRAIL/dulanermin reduces bone lesions and skeletal tumor burden in a model of breast cancer skeletal metastasis. Canc Biol Ther 9:539-550

82. Fizazi K, Carducci M, Smith M et al (2011) Denosumab versus zoledronic acid for treatment of bone metastases in men with castration-resistant prostate cancer: a randomized, double-blind study. Lancet 377:813-822

83. Henry DH, Costa L, Goldwasser F et al (2011) Randomized, double-blind study of denosumab versus zoledronic acid in the treatment of bone metastases in patients with advanced cancer (excluding breast and prostate cancer) or multiple myeloma. J Clin Oncol 29:1125-1132

84. Stopeck AT, Lipton A, Body JJ et al (2010) Denosumab compared with zoledronic acid for the treatment of bone metastases in patients with advanced breast cancer: a randomized, double blind study. J Clin Oncol 28:5132-5139

85. Smith MR, Egerdie B, Hernandez Toriz N et al (2009) Denosumab in men receiving androgen-deprivation therapy for prostate cancer. N Engl J Med 361:745-755

86. Paes FM, Serafini AN (2010) Systemic metabolic radiopharmaceutical therapy in the treatment of metastatic bone pain. Semin Nucl Med 40:89-104

87. Pandit-Taskar N, Batraki M, Divgi CR (2004) Radiopharmaceutical therapy for palliation of bone pain from osseous metastases. J Nucl Med 45:1358-1365

88. Bodei L, Lam M, Chiesa C et al (2008) EANM procedure guideline for treatment of refractory metastatic bone pain. Eur $\mathrm{J}$ Nucl Med Mol Imaging 35:1934-1940

89. Silberstein EB, Taylor AT Jr (2003) EANM procedure guidelines for treatment of refractory metastatic bone pain. Eur J Nucl Med Mol Imaging 30:BP7-BP11

90. Paes FM, Ernani V, Hosein P et al (2011) Radiopharmaceuticals: when and how to use them to treat metastatic bone pain. J Support Oncol 9:197-205 
91. Gkialas I, Iordanidou L, Galanakis I et al (2008) The use of radioisotopes for palliation of metastatic bone pain. J BUON 13:177-183

92. Kurosaka S, Satoh T, Chow C et al (2012) EORTC QLQ-BM22 and QLQ-C30 quality of life scores in patients with painful bone metastases of prostate cancer treated with strontium- 89 radionuclide therapy. Ann Nucl Med doi:10.1007/s12149-012-0598-Z

93. Sciuto R, Tofani A, Festa A et al (1998) Platinum compounds as radiosensitizers in strontium-89 metabolic radiotherapy. Clin Ther 149:43-47

94. Tu SM, Millikan RE, Mengisu B et al (2001) Bone-targeted therapy for advanced androgen-independent carcinoma of the prostate: a randomised phase II trial. Lancet 357:336-341

95. Fizazi K, Beuzeboc P, Lumbroso J et al (2009) Phase II trial of consolidation docetaxel and samarium-153 in patients with bone metastases from castration-resistant prostate cancer. J Clin Oncol 27:2429-2435

96. Morris MJ, Pandit-Tasker N, Carrasquillo J et al (2009) Phase I study of samarium-153 lexidronam with docetaxel in castrationresistant metastatic prostate cancer. J Clin Oncol 27:2436-2442
97. Finlay IG, Mason MD, Shelley M (2005) Radioisotopes for the palliation of metastatic bone cancer: a systematic review. Lancet Oncol 6:392-400

98. Storto G, Klain M, Paone G et al (2006) Combined therapy Sr-89 and zoledronic acid in patients with painful bone metastases. Bone 39:35-41

99. Koutsikos J, Leondi A (2008) Treatment efficacy of combined bisphosphonates and 186Re-HEDP treatment in cancer patients with bone metastases. Eur J Nucl Med Mol Imaging 35:13921393

100. Liang JG, Jiang NY, Du JQ et al (2005) Clinical value of combined therapy with $188 \mathrm{Re}$-HEDP and pamidronate in breast cancer with bone metastasis. Zhonghua Zhong Liu Za Zhi 27:180182

101. Chow E, Nguyen J, Zhang L et al (2012) International field testing of the reliability and validity of the EORTC QLQ-BM22 module to assess health-related quality of life in patients with bone metastases. Cancer 118(5):1457-1465

102. Functional Assessment of Cancer Therapy - Bone Pain. http:// www.facit.org/FACITOrg/Questionnaires. Accessed 20 May 2012 